\title{
Diagnostic Approaches to the Tendon, Ligament and Joint Affections in Bovine with Special Reference to Tendon Elastography
}

\author{
Sheikh Mohd Yaseen Jani", S. K. Mahajan, Pallavi Verma and J. Mohindroo \\ Department of Veterinary Surgery and Radiology, College of Veterinary Science \\ Guru Angad Dev Veterinary and Animal Sciences University, \\ Ludhiana, Punjab 141004, India \\ *Corresponding author
}

\section{A B S T R A C T}

The present study was conducted in 30 clinical cases related to musculoskeletal disorders specific to lameness in bovine, presented to the Department of Veterinary Surgery and Radiology, GADVASU, Ludhiana from August 2015 to

Keywords

Bovine, Joints, Ligament, Strain elastography,

Tendon,

Ultrasonography

Article Info

Accepted:

12 March 2020

Available Online:

10 April 2020 July 2017. The animals suffering from musculoskeletal abnormalities specific to lameness were divided into five major groups viz. Group-1 tendon rupture and partial tear $(n=6)$, Group 2-tendon and tendon sheath affections $(n=18)$, Group 3affection of joint $(n=1)$, Group 4-abscess $(n=2)$ and Group 5-bone affections $(n=3)$. Detailed signalment, history, gait abnormalities, physical examination, flexion test and hematobiochemical parameters were recorded and compared to the values of normal animals. A 7-12 MHz transducer was used for evaluation of the tendons, ligaments and joints in bovine. Ultrasonography, elastography and radiography (if required) were conducted on all the animals. It was concluded that ultrasonography is important for the diagnosis of tendon and tendon sheath affection, hematoma, abscess and joint affection in bovine. Elastography was used to assess the mechanical properties of tendons qualitatively and quantitatively. It allowed the detection of tissue strain and provided valuable mechanical information about bovine tendon injuries.

\section{Introduction}

Skeletal and articular disorders are by far the most common cause of musculoskeletal injuries in large animals and are a major source of debilitating pain, economic loss and loss of athleticism. Bovine are more susceptible to the tendon, ligament and joint affections because of their heavy body weight. Lameness in cattle is a consequence of diseases of joints and ligaments (47\%), tendons $(21 \%)$, muscles $(9 \%)$, bones $(7 \%)$, nerves $(7 \%)$ and other tissues including skin (10\%) (Baggott and Russell 1981). The first step towards diagnosis of lameness is obtaining detailed history of the animal. 
Visual examination enables to evaluate swelling or wound on the body of the animal. The area of lesion can be located by observing the gait of the animal (Stashak 1987). To locate the exact location of origin of lameness extension and flexion tests are performed (Ramey 1997). Radiography remains the cornerstone of diagnostic imaging technique for the evaluation of the musculoskeletal disorders.

After localization of lameness by means of physical examination, survey radiographs can quickly and accurately provide morphologic characterization of bone and soft tissue abnormalities which concurrently lead to formation of a definitive or differential diagnosis (Vallance et al., 2012). Ultrasonography is ideal for the evaluation of musculoskeletal disorders because these are often associated with extensive soft tissue swelling and inflammatory exudation.

Ultrasonography also improves the likelihood of a definitive diagnosis of tendon, ligament or joint affection. The advantage that ultrasonography has over radiography is the fact that a three-dimensional structure is projected onto a two-dimensional plate in radiography which leads to the major disadvantage of superimposition of bony structures and lack of differentiation of soft tissues (Kraft and Gavin 2001).

Elastography is an in situ method that can be used to assess the mechanical properties of soft tissue qualitatively and quantitatively through ultrasonographic imaging techniques (Klauser et al., 2014). Drakonaki et al., (2009) used real time freehand ultrasound elastography to evaluate normal Achilles tendon and to describe its elastographic appearances. It also allows detection and measurement of tissue strain, and may provide valuable mechanical information about equine tendon injuries.

\section{Materials and Methods}

The present study was conducted on clinical cases of musculoskeletal disorders related to lameness in bovine presented to the department of veterinary surgery and radiology, GADVASU, Ludhiana from February 2015 to June 2017. 30 bovine presented for various tendons, ligament and joint affection were included in the study. The animals were grouped according to the condition diagnosed (Table 1). Detailed history including duration of lameness, type of injury, visual examination, palpation and flexion tests were done. On the basis of visual examination, scoring of lameness was done as proposed by Sprecher et al., (1997) (Table 2).

The hematobiochemical parameters like $\mathrm{Hb}$ $(\mathrm{g} / \mathrm{dL})$, TLC (per $\mu \mathrm{l})$, DLC $(\%)$, creatinine kinase (U/L) and serum lactate dehydrogenase (mmol/L) were recorded as well. The cases suspected for pathological condition related to lameness were subjected to radiography. Ultrasonography was carried out using a 7- 12 $\mathrm{MHz}$ convex probe of GE Logiq 3 BT expert ultrasound machine or concept / MCV veterinary ultrasound scanner (Dynamic Imaging Co., Scotland, U.K.), in real time Bmode with a $5 \mathrm{MHz}$ linear and $3.5 \mathrm{MHz}$ micro convex transducer with the limb in weight bearing position.

\section{Strain elastography (SE)}

Strain elastography (SE) was performed with a 7-12 MHz linear transducer for tendons. A depth of 3-6 cm was used for scanning the tendons. Unlike ultrasonography, in elastography, it was important to keep the target limb relaxed during elastographic examination. Multiple attempts were allowed to produce the elastographic images and the same were recorded when either elastographic alterations were detected in areas of known lesions or after several repeated attempts did 
not produce elastographic alterations. If signal could not be obtained, the size of the elastographic box was decreased until elastographic signal could be produced. Transverse and longitudinal images of the tendons were obtained and a qualitative assessment of images was performed using a modified version of grading system proposed by Itoh et al., (2006) for elastography score (ES) known as Tsukuba classification for human breast examination.

In the Tsukuba classification, an ES of 1, 2 or 3 were considered as benign and lesions categorized as ES 4 and 5 were suspicious for cancer (Wojeinski et al., 2013). Since malignant tumor is considered more invasive and comparatively harder in consistency as compared to benign tumor, a higher ES was given. In case of tendon elastography, tendons are comparatively harder when compared to breast tissue in humans but during injury there is reduction in the elasticity of the tendons as softness at the injury site develops. The scoring for tendon was made such that the normal tendons which are harder were given higher ES and the affected tendon which were relatively less stiff were given lower score. This was the opposite of Tsukuba classification. Therefore Tsukuba classification did not hold good for tendons and was extrapolated by making modification in scoring.

\section{Results and Discussion}

Among bovine, 11 animals were male and 19 animals were female. The maximum age (in years) of bovine suffering from lameness was seen in group 2 (tendinitis and tendon sheath affections, $\mathrm{n}=18$ ) which ranged from 6 months to 10 years with a mean \pm SE of 6.02 \pm 0.73 and minimum age (in years) was seen in group 5 (bone affections, $n=3$ ) which ranged from 6 months to 3 years with a mean $\pm \mathrm{SE} 4.66 \pm 0.72$. Out of 30 cases reported among bovine, $50 \%(15 / 30)$ cases were reported among the milch type cattle which may be due to presence of high number of milch bovine in the region. The bovine belonging to group 1 with tendon rupture and partial tears, had shortest duration of illness i.e. $6.66 \pm 2.52$ days. The maximum duration of illness was seen in group 5 (bone affection) with a mean \pm SE $70 \pm 20.02$ days, which may be due to slow progression of the illness or inability to diagnose the condition during early stages.

The bovine suffering from the tendon rupture and partial tears (group 1) were given the highest lameness score with the mean value of $4.83 \pm 0.16$ as the animals were not able to bear weight on the affected limb. Anderson and Jean (1996) also reported severe lameness in animals with tendon rupture. The high lameness score in bovine was given in the bone affection (group 5) as well i.e. a mean value of $4.33 \pm 0.66$, as the animal had difficulty in bearing weight on the affected limb (Kofler, 1996). The flexion tests were positive in $83 \%(25 / 30)$ cases of bovine suffering from musculoskeletal affections related to lameness. The flexion tests only provided information about the pain in the associated joints but failed to pin point the exact location of the site of lameness. Ramey (1997) also suggested that flexion tests cannot detect the site of lameness and reliance on these tests to predict future of lameness was unwise.

The value of hemoglobin was within the normal range in all animals, irrespective of the group. The observation of hemoglobin values in the present study were in accordance to earlier workers, who also reported that the hemoglobin values were within the normal in animals suffering from various musculoskeletal disorders related to lameness (Pilsworth and Head, 2001). Elevated mean value of TLC was observed in bovine (24522 
\pm 5.01 per $\mu \mathrm{L}$ ) suffering from abscess. Similar findings were reported by Smeets et al., 1990. Mean value of TLC remained high in all the groups in bovine. All the animals irrespective of the group suffered from neutrophilia and consequently lymphopenia which was more pronounced in case of abscess (bovine). These findings were in agreement with Coles (1974). The values of the serum lactate were high in all the groups in bovine. The maximum value of serum lactate was found in bovine in the abscess group (5.80 \pm 0.20 mmol/L). Spriet et al., (2000) also suggested the increased serum lactate values associated with musculoskeletal injuries related to lameness. The values of serum creatinine kinase were high in all the groups except in group 3 and group 4 among bovine. The values of serum creatinine kinase were highest in group 2 among bovine (1153.28 \pm 36.37 U/L). These findings were in agreement with Valberg (2009).

\section{Radiography}

In bovine, radiography was done either in standing animal or after restraining the animal in lateral recumbency. In group $1(n=6)$, the radiographs were taken in all bovine to record the possible bone injury along with severed tendons. Among 6 bovine, 2 were suffering from SDF and GC tendon tear. Radiographically it was visible as subcutaneous infiltration of tissue with air pockets that lead to increase of contrast in the target area and made tendon rupture appreciable radiographically (Fig. 1). In the other animals belonging to this group no radiographic abnormalities were appreciable. In group $2(n=18)$, bovine suffering from the tendinitis and tendon sheath affections a total of 18 cases were studied out of which 13 suffered from tendinitis and desmitis and rest suffered from tendon sheath affections. SDF and DDF tendinitis were most commonly recorded in this group. No radiographic abnormality was seen in the 13 cases suffering from SDF and DDF tendinitis except mild soft tissue swelling which was evident radiographically. In 4 cases suffering from tendon sheath affection no significant radiographic changes were seen except in one in which there was severe periosteal reaction on the dorsal surface of metatarsal along with soft tissue swelling and gas pocket formation around the hock joint (Heppelmann et al., 2009) (Fig. 2). In case of bovine (group 3), septic arthritis of the elbow joint was evident and the radiographic changes observed included destruction of the structural anatomy of the elbow joint with severe periosteal reactions on the dorsal aspect of the joint and presence of multiple osteophytes (Fig. 3). Radiographically if there is reduction of joint space, formation of osteophytes and periosteal reactions either on proximal or distal margin of the bones will characterize joint affection (Riley and Farrow, 1998). In group 5 (bone affection), two cases were suffering from chronic contracted tendon of forelimb which led to changes in the elbow joint leading to severe osteophyte formation and periosteal reaction. In one case of bovine, there was soft tissue reaction around the stifle joint which could have resulted from the ligament injury or an old fracture of tibial tuberosity (Fig. 4). Vanderperren et al., 2012 also described similar changes on the dorsal aspect of the metacarpus which was associated with the injury in the past.

\section{Ultrasonography}

A 7-12 MHz linear transducer was used to perform ultrasonography of tendons, ligaments and joints. A depth of 3-6 cm was used for scanning of the target tissue. The scanning procedure was similar to part I of the study already described for tendons and ligaments. A 3.5 MHz convex transducer was used for scanning deeper structures like abscess and hematoma. 
In bovine with tendon rupture and partial tears (group 1), ultrasonography was performed to evaluate the tendon for any rupture, condition of the edges and gap assessment. In case of bovine, a total of 6 cases were recorded in this category out of which 4 animals had SDF and DDF rupture in the region of metatarsal and rest 2 animals had GC rupture. Among 4 animals with SDF and DDF tear, the tendons appeared to have lost the fiber orientation and showed the presence of large anechoic areas (Fig. 5). Avella 2009 also reported that the area of fiber damage occurs in the center of the affected tendon and appears as a hypoechoic or an anechoic area. The tendon tear had also resulted in the formation of a small gap in between the tendons. Seliem et al., (2002) reported that ultrasonographic examination of ruptured digital flexor tendons was characterized by presence of the ruptured echogenic tendinous segments and the cut area appeared anechoic in between with peritendinous anechoic fluid.

It was also reported that in longitudinal view the tendon tear showed lack of fiber arrangement but this fiber tearing could be multiple, showed hypoechoic to an echoic area with slight loss of fibers alignment at the dorsal aspect of the tendon. In case of GC tear, the fibers of the GC were echolucent but with sharp edges which may be due to misalignment of GC fibers due to injury. There was also presence of anechoic inflammatory fluid around the tendon (Fig. 6).

This findings were in accordance with Duenwald et al., (2011) who reported that the alteration in tendon echogenicity reflects changes in the cellular and extracellular composition of injured/ severed tendons along with the repair tissue, it ranged from a barely perceptible decrease in echogenicity with mild loss of fiber pattern seen with fiber rupture accompanied with hemorrhage or with serious fluid accumulation and edema.
In animals of group 2 with tendon and tendon sheath affections, ultrasonography was performed to evaluate the echotexture of the tendons and the content of tendon sheaths. Among 18 bovines in this group, 12 were suffering from DDF tendinitis in the lateral digit of pastern joint. DDF tendinitis was marked by decrease in the echogenicity of the tendon coupled with multiple anechoic areas in the tendon (Fig. 7). Barr et al., 1995 reported that the tendinitis was ultrasonographically represented by the small, distinct often circular, focal hypoechoic areas within the tendon and the increase in the size of the tendons. In one bovine SL desmitis was observed. The suspensory ligament injury was recorded at the mid carpal region on palmar aspect. SL desmitis was characterized by increase in the thickness of the SL along with misalignment of the fibers with decreased echogenicity and presence of multiple anechoic areas in the target area. The underlying metacarpal did not show any bone abnormalities owing to acute nature of insult. SL branch injuries were difficult to detect but unusual swelling around the branch and pain on palpation can be considered as pointers for examination (Fig. 8). These findings were in agreement with Rantanen (2008). Smith (2008) reported about the ultrasonographic features of injury to SL which include enlargement of the SL, poor definition to the margins, single or multiple poorly defined focal areas of hypoechoicity, diffuse hypoechoicity and irregularity of the palmar surface of the metacarpus/metatarsus which is indicative of enthesiophytosis.

Three bovine cases which were suffering from chronic lameness were reportedly having tendon sheath affection. In this case, the palmar aspect of SDF was ill defined and was incorporated into a chronic, fibrous tissue mass forming extensive adhesions due to digital tendon sheath effusion. The palmar surface of SDF was damaged and was 
incorporated into the reaction. Restrictive SDF tendon adhesions were evident when the target area was imaged dynamically during flexion and extension. The distension of the tendon sheath was represented by the enlargement of size of the tendon sheath by Minshall and Wright 2012 who also reported that he echogenicity of the content of the tendon sheaths was not constant as it relied on the amount of cellular content. Rest two bovine were reported with SDF tendinitis in mid metatarsal region. In SDF tendinitis, there was increase in the tendon size along with present of anechoic fluid around the tendon. SDF tendinitis also showed separation of the core fibers of the tendon along with increase in the tendon gap (Belt et al., 2011) (Fig. 9). In group 3 with affections of the joint, ultrasonography was done to evaluate the distension of the joint pouches. Among bovine, one case had joint affection of the hock joint.

It was associated with the soft tissue swelling and presence of fluid around the affected area. Heppelmann et al., 2009 also described that the echoic content was seen in joints with septic arthritis and it was used to differentiate between the septic and non-septic condition of the joint. Non-septic arthritis of the hock joint was evident with presence of joint pouches with echoic content were seen on the medial and lateral compartments of the tibiotarsal joint (Fig. 10). Similar observations were reported by Kofler (2009).

In group 4 and 5 with abscess and hematoma respectively, ultrasonography was done to evaluate the echogenicity of the content and involvement of the underlying structures. Among bovine, 2 cases were suffering from abscess. The abscess was located on the lateral and medial aspect of hock joint. The abscess on the lateral aspect hock joint was oozing pus in one case. During ultrasonography, the abscess cavity was clearly marked with a chalk so as to demarcate the extent of the cavity which would facilitate in drainage of the abscess cavity through the dependent part. In animals of group 5 with bone affections, subtle changes on the surface of the bone were visualized. Ten animals were included in this group with 3 bovine and 7 equine patients. Among bovine, one animal had an old fracture of tibial tuberosity and the rest two were chronic cases of contracture of fetlock and pastern joints. In bovine with old fracture of tibial tuberosity, periosteal reaction was visible over the plantar surface from mid distal tibial bone (Vanderperren et al., 2012).

\section{Strain elastography}

Strain elastography (SE) was performed with a 7-12 MHz linear transducer for tendons. A depth of 3-6 cm was used for scanning the tendons. The bovine belonging to group 1 (tendon rupture and partial tear) involved a total of 6 animals under the study. The elastography score (ES) ranged from 1 to 2 in this group with a mean \pm SE value of $1.33 \pm$ 0.21 . The lowest ES was seen in cases of Gastrocnemius (GC) tendon rupture ( $\mathrm{ES}=1)$. Complete GC tear was confirmed on B-mode ultrasonography and on elastography the hypoechoic region in the tendon and loss of fiber orientation of the GC tendon were appreciable on elastogram as well towards the softer side (green and red mosaic) (Fig. 11). Similar observations were made by Lustgarten et al., 2015 who observed a correlation between the hypoechogenecity of lesions on gray-scale ultrasonography and softness on elastography with more hypoechoic lesions appearing softer and more hyperechoic lesions harder.

A total of 18 cases were studied under group 2 (tendinitis and tendon sheath affections) in bovine. The ES ranged from 1 to 3 in this group with a mean $\pm \mathrm{SE}$ value of $2.05 \pm 0.12$. 
In this group, a total of 18 cases were recorded with 12 suffering from DDF tendinitis in the lateral digit of pastern joint, 1 with SL desmitis, 3 with tendon sheath affection (chronic lameness) and rest two cases were presented with SDF tendinitis. In DDF tendinitis, in addition to the location of the lesions revealed by B-mode ultrasonography, elastography made it possible to minutely analyze the region of interest (ROI) and also contributed to better understanding of the tendon injury by providing a platform for real time comparison between different regions of a tendon or between different tendons at the same time. Elastography also proved helpful in differentiating between the acute and chronic injury within a tendon which was not possible otherwise (Fig. 12 and 13). The differentiation was possible because the stiffness of the acute lesion increases as the time progresses and this is picked up by the software of the elastogram. Drakonaki et al., (2009) reported the findings of injured Achilles tendons in people in which areas of fiber disruption and hematoma formation exhibited increased softness when compared with normal tendons.

Table.1 Distribution of various musculoskeletal disorders in bovine

\begin{tabular}{|l|l|l|}
\hline Group & Disorders & Bovine \\
\hline $\mathbf{1}$ & Tendon rupture and partial tear & 6 \\
\hline $\mathbf{2}$ & Tendinitis and tendon sheath affections & 18 \\
\hline $\mathbf{3}$ & Affections of joints & 1 \\
\hline $\mathbf{4}$ & Abscess & 2 \\
\hline $\mathbf{5}$ & Bone affections (other than long bone fractures) & 3 \\
\hline
\end{tabular}

Table.2 Lameness grading system in bovine (Sprecher et al., 1997)

\begin{tabular}{|l|l|l|}
\hline $\begin{array}{l}\text { Lameness } \\
\text { score }\end{array}$ & $\begin{array}{l}\text { Clinical } \\
\text { description }\end{array}$ & Assessment criteria \\
\hline $\mathbf{1}$ & Normal & $\begin{array}{l}\text { Stands and walks with a level back posture; normal } \\
\text { gait. }\end{array}$ \\
\hline $\mathbf{2}$ & Mildly lane & $\begin{array}{l}\text { Stands and walks with a level back posture, Arched } \\
\text { back posture while walking; normal gait. }\end{array}$ \\
\hline $\mathbf{3}$ & $\begin{array}{l}\text { Moderately } \\
\text { lame }\end{array}$ & $\begin{array}{l}\text { Arched back posture while standing and walking; } \\
\text { short- striding gait. }\end{array}$ \\
\hline $\mathbf{4}$ & Lame & $\begin{array}{l}\text { Arched back posture is always evident: gait described } \\
\text { as one deliberated step at a time. }\end{array}$ \\
\hline $\mathbf{5}$ & Severely lame & $\begin{array}{l}\text { Inability or extreme reluctant to bear weight on one } \\
\text { or more of her limbs. }\end{array}$ \\
\hline
\end{tabular}




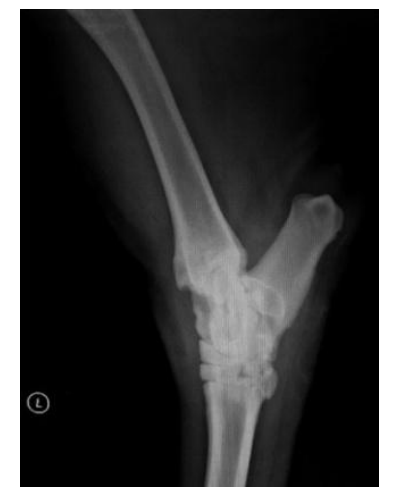

Fig.1 Lateral radiograph of tibial region showing gas pocket formation around severed tendon

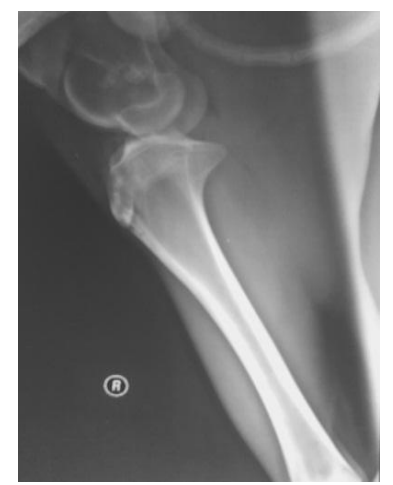

Fig.4 Lateral radiograph of the stifle joint showing soft tissue reaction

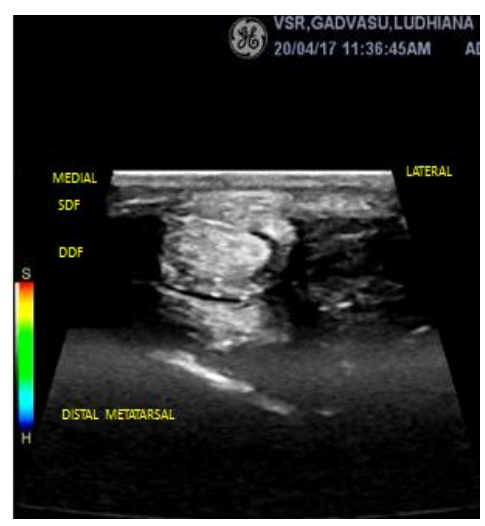

Fig.7 Transverse ultrasonogram of distal metatarsal region of bovine showing DDF tendinitis

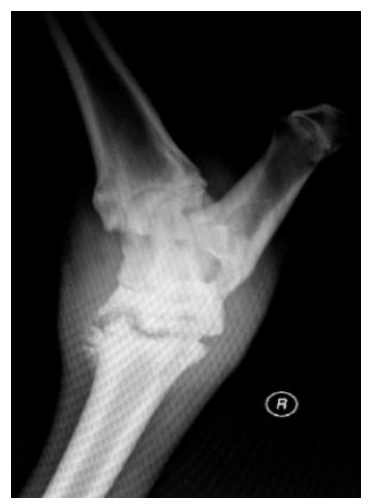

Fig.2 Lateral radiograph of hock joint showing periosteal reaction on the dorsal surface of metatarsal

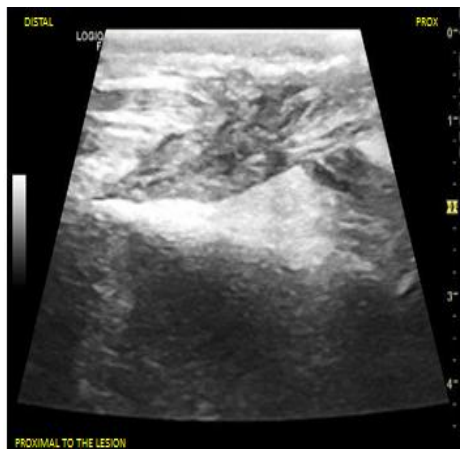

Fig.5 Longitudinal

ultrasonogram of mid metatarsal region showing complete severing of SDF and

\section{DDF tendons}

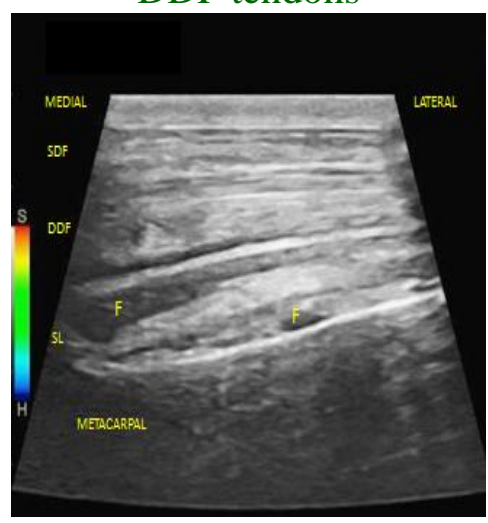

Fig.8 Longitudinal ultrasonogram of midmetatarsal region showing SL desmitis

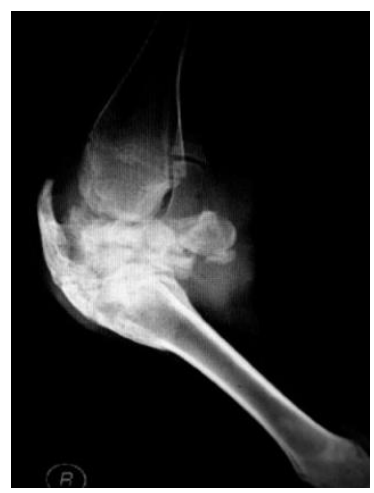

Fig.3 Lateral radiograph of carpal joint showing osteophyte formation

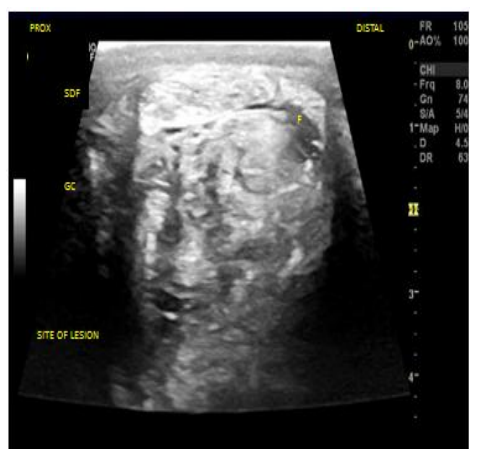

Fig.6 Transverse ultrasonogram at the level of distal aspect of tibial region revealing $\mathrm{GC}$ tendon tear

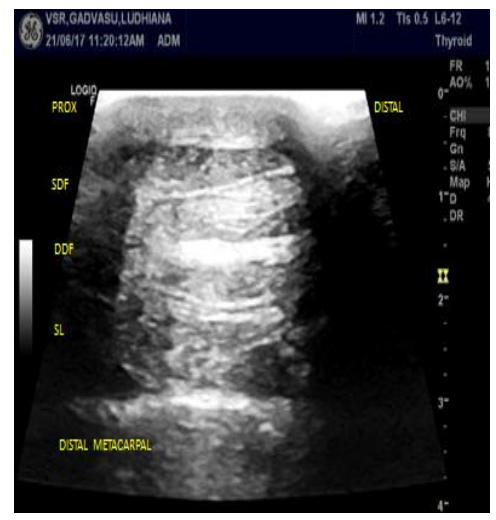

Fig.9 Transverse ultrasonogram of distal metacarpal region showing SDF tendinitis 


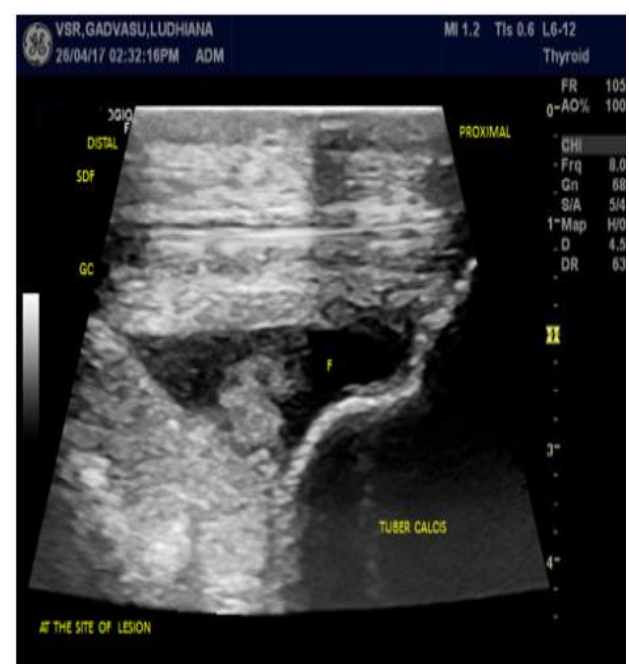

Fig.10 Longitudinal ultrasonogram of distal tibial region with distension of hock joint

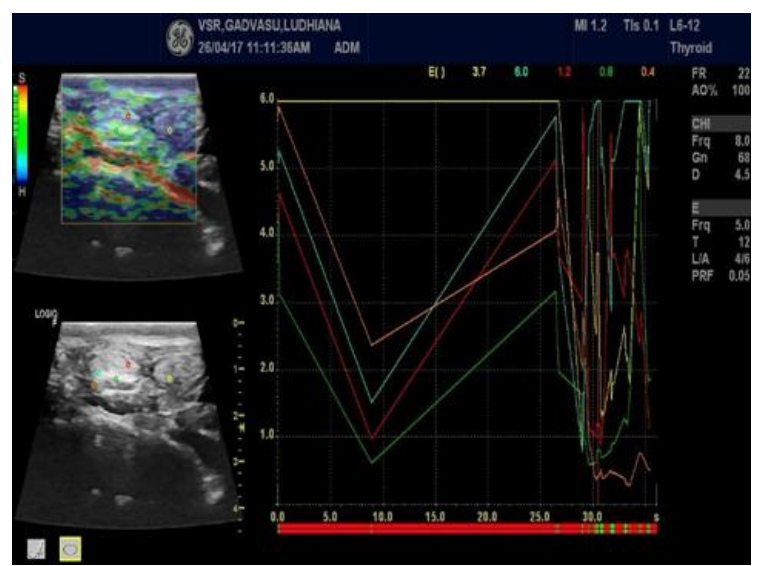

Fig.12 Transverse SE of distal metatarsal region $(\mathrm{ES}=2)$ showing DDF tendinitis and chronicity of lesion

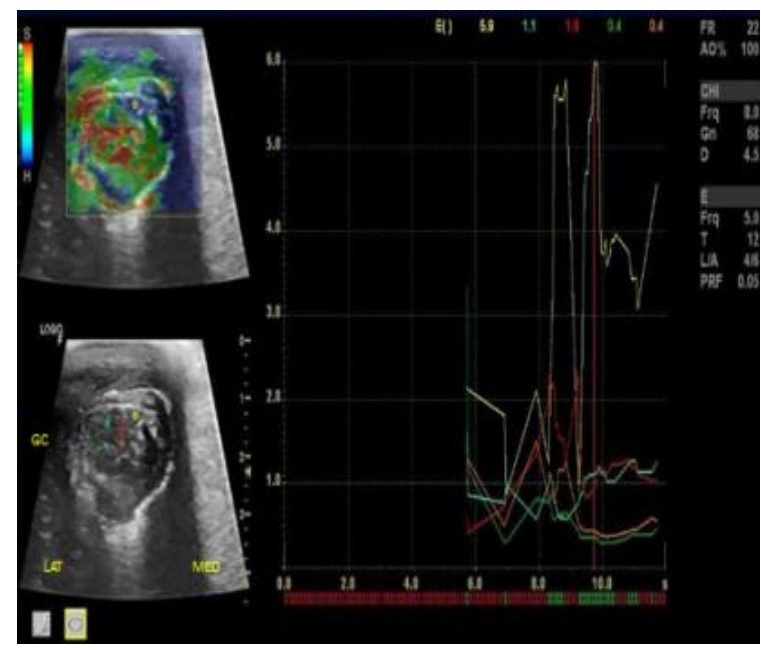

Fig.11 Transverse $\mathrm{SE}$ of bovine $(\mathrm{ES}=1)$ showing complete GC tear

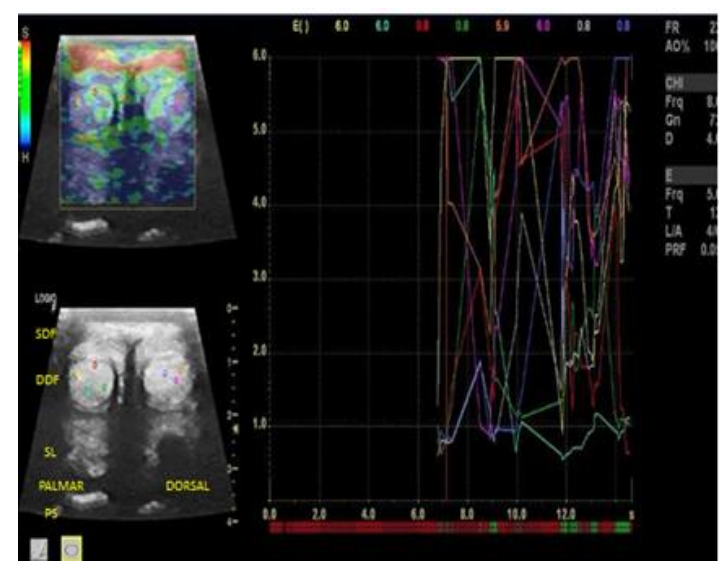

Fig.13 Transverse SE of distal metatarsal $(E S=1)$ of pastern region showing SDF tendinitis

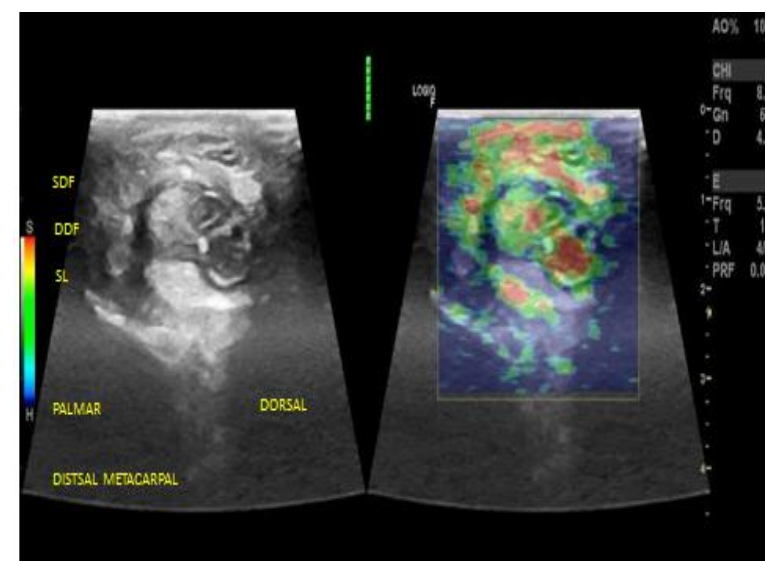

Fig.14 Transverse $\mathrm{SE}$ of $(\mathrm{ES}=2.05 \pm 0.12)$ distal metacarpal region showing tendon sheath distension 
Additionally, Thorpe et al., (2010) reported that the progressive increase in stiffness of acute lesions as time progressed was expected as collagen deposition occurs over time adding to the stiffness of the lesions. In one case of SL desmitis observed at the level of distal metatarsal elastogram was more accurate in revealing that the SL of lateral aspect was more affected than the medial side which was not possible to determine with routine B-mode ultrasonography. Klauser et al., (2013) also reported that correlating elastography with histology of Achilles tendons concluded that elastography was useful to detect degeneration and maybe more sensitive than gray-scale ultrasonography (Fig. 14).

DDF tendinitis was most common affection of tendons seen among bovine and was most common successfully diagnosed condition with B-mode ultrasonography. A 7-12 MHz linear transducer and a tissue depth of 3-4 cm was suitable for evaluation of tendons, ligaments and joints in large animals. Ultrasonography was helpful in assessment of complete and partial tendon tears and was reliable in the diagnosis of tendon and tendon sheath affections along with hematoma, abscess and joint affections. Elastography proved as a useful tool in not only determining the extent of damage sustained by a tendon but also helped in differentiation between acute and chronic lesions. Elastography helped in detecting degeneration in the tendon and proved more sensitive than B- mode ultrasonography.

\section{Acknowledgement}

The authors thank Indian Council of Agricultural Research (ICAR) for providing funds under the scheme All India Network Program on Diagnostic Imaging and Management of Surgical Conditions in Animals (AINP-DIMSCA).

\section{References}

Anderson, D. E., and Jean. 1996. Traumatic flexor tendon injuries in 27 cattle. Veterinary surgery. 25, 320-326.

Avella, C. S., Ely, E. R., Verheyen, K. L. P., Price, J. S., Wood, J. L. N., and Smith, R. K. W. 2009. Ultrasonogrprahic assessment of the superficial digital flexor tendons of National Hunt racehorses in training over two racing seasons. Equine Veterinary Journal. 41(5), 449-454.

Baggott, D. G., and Russell, A. M. 1981. Lameness in cattle. British Veterinary Journal. 137, 113-132.

Barr, A. R., Dyson, S. J., Barr, F. J., and O'Brien, J. K. 1995. Tendonitis of the deep digital flexor tendon in the distal metacarpal/metatarsal region associated with tenosynovitis of the digital sheath in the horse. Equine Veterinary Journal. 27, 348-355.

Belt, A. J., Becker, C. K. and Dik, K. J. 2011. Desmitis of the accessory ligament of the deep digital flexor tendon in the horses: Clinical and Ultrasonographic features. A report of 24 cases. Zentralbl Veterinarmed A 40.

Coles, E. H. 1974. Veterinary Clinical Pathology. $\quad 2^{\text {nd }} \quad$ Edn. $\quad$ Saunders Publishers, Philadelphia, USA.

Drakonaki, E. E., Allen, G. M., and Wilson, D. J. 2009. Real-time ultrasound elastography of the normal Achilles tendon: reproducibility and pattern description. Clinical Radiology. 64, 1196-1202.

Duenwald, S., Kobayashi, H., Frisch, K., Lakes, R., and Vanderby, R. 2011. Ultrasound echo is related to stress and strain in tendon. Journal Biomechistry. 44, 424-429.

Heppelmann, M., Kofler, J., Meyer, H., Rehage, J., and Starke, A. 2009. Advances in surgical treatment of septic 
arthritis of the distal interphalangeal joint in cattle: A review. Veterinary Journal. 82, 162-175.

Itoh, A., Ueno, E., and Tohno, E. 2006. Breast disease: clinical application of US elastography for diagnosis. Radiology. 239 (2), 341-350.

Klauser, A., Miyamoto, H., and Tamegger, M. 2013. Achilles tendon assessed with sonoelastography: histologic agreement. Radiology. 267, 837-842.

Klauser, A. S., Miyamoto, H., BellmannWeiler, R., Feuchtner, G. M., Wick, M. C., and Jaschke, W. R. 2014. Sonography: musculoskeletal applications. Radiology. 272, 622-633.

Kofler, J. 1996. Arthrosonography- the use of diagnostic ultrasound in septic and traumatic arthritis in cattle- A retrospective study of 25 patients. British Veterinary Journal. 152, 683698.

Kofler, J. 2009. Ultrasonography as a diagnostic aid in bovine musculoskeletal disorders. The Veterinary Clinics North America Food Animal Practice. 25, 687-731.

Kraft, S. L. and Gavin, P. 2001. Physical principles and technical considerations for equine computed tomography and magnetic resonance imaging. Veterinary Clinics of North America: Equine Pracitce.17, 115-130.

Lustgarten, M., Redding, W. R., Labens, R., Morgan, M., Davis, W., and Seiler, G. S. 2014. Elastographiccharacterstics of the metacarpal tendons in horses without clinical evidence of tendon injury. Veterinary Radiology and Ultrasound. 55, 92-101.

Minshall, G. H., and Wright, J. M. 2012. Tenosynovitis of carpal sheath of digital flexor tendons associated with tears of radial head and deep digitak flexor observation in 11 horses. Equine Veterinary Journal. 44, 76-80.
Pilsworth, R. C., and Head, M. J. 2001. A study of 10 cases of focal peritarsal infection as a cause of severe lameness in the thoroughbred racehorse: Clinical signs, differential diagnosis, treatment and outcome. Equine Veterinary Journal. 33, 366-370.

Ramey, D. W. 1997. Prospective Evaluation of Forelimb Flexion tests in Practice: Clinical Response, Radiographic Correlations and Predictive Value for Future Lameness. Proceedings of the $43^{\text {rd }}$ Annual Convention of American Association of Equine Practitioners. pp 116-20.Tenessee.

Rantanen, N. W. 2008. Musculoskeletal Ultrasonography. Compendium Equine, pp. 356-364.

Riley, C. B. and Farrow, C. S. 1998. Partial carpal arthrodies in a calf with chronic infectious arthritis of the carpaus and osteomyelitis of the carpal and metacarpal bones. Canadian Veterinary Journal. 39, 438-441.

Seliem, M. A., El-Sayad, G. A., Kamel, A. M. 2002.Ultrasonographic diagnosis of some tendons and ligament affections in equine limbs. Journal Egypt Veterinary Association. 62, 323-338.

Smeets, J. F. M., Odink, J., Visser, I. J. R., Schoen, E. D., and Snijders, J. M. A. 1990. Haematology and blood chemistry for predicting abscesses and other abnormalities in slaughtered pigs. Veterinary Quarterly. 12, 146-151.

Smith, R. K. W. 2008.Tendon and Ligament. AAEP Proceedings. 54, 475-501.

Sprecher, D. J., Hostetler, D. E., and Kaneene, J. B. 1997. A lameness scoring system that uses posture and gait to predict dairy cattle reproductive performance. Theriogenology. 47, 1179-1187.

Spriet, L. L., Howlett, R. A., and Heigenhauser, G. J. 2000. An enzymatic approach to lactate production inhuman 
skeletal muscle during exercise. Medicine and Science in Sports and Exercise. 32,756-763.

Stashak, T. S. 1987. Lameness. In: Stashak T $S$ (Ed.) Adams' Lameness in Horses. $4^{\text {th }}$ Edn. Philadelphia: Lea \& Febiger, pp. 675.

Thorpe, C. T., Clegg, P. D., and Birch, H. L. 2010. A review of tendon injury: why is the equine superficial digital flexor tendon most at risk? Equine Veterinary Journal. 42, 174-180.

Valberg, S. J. 2009. Approach the horse with a suspected myopathy. In Proceedings of the $11^{\text {th }}$ International Congress of the World Equine Veterinary Association, pp. 3-15.

Vallance, S. M., Bell, R. J.,Spriet, M., Kass, P. H., and Puchalski, S. M. 2012. Comparisons of computed tonography, contrast enhanced computed tomography and standing low-field magnetic resonance imaging in horses with lameness localized to the foot. Part1: Anatomic visualization scores. Equine Veterinary Journal. 44, 51-56.

Vanderperren, K., Gielen, I., Caelenberg, A. V., Vekens, E. V., Raes, E. V., Hauspie, S., Bree, H., and Saunders, J. H. 2015. Ultrasonographic appearance of bony abnormalities at the dorsal aspect of the fetlock joint in geriatric cadaver horses. The Veterinary Journal. 193, 129-134.

Wojeinski, S., Boehme, E., Farrokh, A., Soergel, P., Degenhardt, F., and Hillemanns, P. 2013. Ultrasound realtime elastography can predict malignancy in BI-RADS (R) - US 3 lesions.BMC Cancer. 13, 159.

\section{How to cite this article:}

Sheikh Mohd Yaseen Jani, S. K. Mahajan, Pallavi Verma and Mohindroo, J. 2020. Diagnostic Approaches to the Tendon, Ligament and Joint Affections in Bovine with Special Reference to Tendon Elastography. Int.J.Curr.Microbiol.App.Sci. 9(04): 1464-1475.

doi: https://doi.org/10.20546/ijcmas.2020.904.172 